\title{
Characteristics of GaInNAsSb/GaAs VCSELs Operating Near 1.55um
}

\author{
James A. Gupta ${ }^{a}$, Stephane Calvez ${ }^{b}$, Nicholas Laurand ${ }^{b}$, Jelmer Weda ${ }^{b}$, David Burns ${ }^{b}$, Daniel \\ Poitras $^{a}$, Geof C. Aers ${ }^{a}$ and Martin D. Dawson ${ }^{b}$, \\ ${ }^{a}$ Institute for Microstructural Sciences, National Research Council of Canada, Ottawa, Canada \\ K1A 0R6; \\ ${ }^{b}$ Institute of Photonics, University of Strathclyde, Wolfson Centre, 106 Rottenrow, Glasgow, \\ G4 0NW, Scotland, UK
}

\begin{abstract}
A detailed study of the high-power pulsed operation of C-band optically-pumped GaInNAsSb vertical cavity surface emitting lasers is reported. The devices employ a resonant periodic gain structure grown by molecular beam epitaxy on a GaAs substrate with a 31-pair GaAs/AlAs bottom distributed Bragg reflector and a 4- $\lambda$, GaAsbased resonant cavity containing 10 GaInNAsSb quantum wells distributed among the 7 antinodes of the electric field. A dual-pump-band $\mathrm{SiO}_{2} / \mathrm{TiO}_{2}$ dielectric top mirror allows efficient optical pumping via low reflectivities at $808 \mathrm{~nm}$ and $1064 \mathrm{~nm}$ while providing very high reflectivity at the $1.55 \mu \mathrm{m}$ target emission wavelength. The laser characteristics were evaluated using both a Q-switched Nd:YAG $1064 \mathrm{~nm}$ pump and a $20 \mathrm{~W}$-peak $180 \mathrm{~ns}-$ pulsed $850 \mathrm{~nm}$ diode laser. The importance of the gain-cavity detuning was evident from time-dependent spectral measurements of laser material subjected to post-growth annealing at different temperatures between 725 and $775^{\circ} \mathrm{C}$. The highest annealing temperature produces the largest blue shift of the gain peak relative to the cavity resonance, resulting in the best power transfer characteristics as well as reduced temperature sensitivity.
\end{abstract}

Keywords: GaInNAsSb, InGaAsNSb, VCSEL, vertical cavity surface emitting laser, disk laser, spectroscopy

\section{INTRODUCTION}

Several materials systems have emerged as potential candidates for vertical cavity surface emitting lasers (VCSELs) in the telecommunications C-band, including In AlGaAs/InP, ${ }^{1,2} \mathrm{AlGaAsSb} / \mathrm{InP},{ }^{3}$ metamorphic GaSbbased devices on GaAs substrates, ${ }^{4}$ and coherently-strained GaAs-based devices employing dilute nitride active regions. ${ }^{5-7}$ Compared with the other alternatives, GaAs-based devices benefit from the high index contrast of GaAs/AlGaAs distributed Bragg reflectors which are nearly lattice-matched to GaAs; robustness of GaAs substrates and maturity of processing. Excellent results have also been obtained by wafer bonding InAlGaAs/InP active regions with GaAs/AlGaAs mirrors, ${ }^{8}$ though monolithically-grown GaAs-based devices have the obvious benefit of processing simplicity. For such devices, the most promising active regions employ quinary GaInNAsSb quantum wells (QWs), which are already beginning to show good performance in $1.55 \mu \mathrm{m}$ edge-emitting lasers, ${ }^{9-15}$ and excellent performance in $1.3 \mu \mathrm{m}$ VCSELs. ${ }^{16}$ Previously we reported the first C-band optically-pumped GaInNAsSb VCSELs at $1547 \mathrm{~nm}\left(20^{\circ} \mathrm{C}\right) .{ }^{5}$ Electrically-pumped GaInNAsSb VCSELs have so far exhibited operation in pulsed mode at $1460 \mathrm{~nm}\left(0^{\circ} \mathrm{C}\right)^{6}$ and at $1534 \mathrm{~nm}\left(-48^{\circ} \mathrm{C}\right) .{ }^{7}$ Another exciting possibility for these materials is in the development of vertical external cavity surface emitting lasers (VECSELs), which are also known as semiconductor disk lasers. These optically-pumped devices have the potential to challenge traditional solid-state lasers in terms of tunability as well as output power. ${ }^{17}$ Dilute nitride devices (GaInNAs) have already exhibited good performance near $1.3 \mu \mathrm{m}$, reinforcing this concept.

Commercial VCSELs in the O-band often employ GaInNAs QWs ${ }^{18}$ which have attractive properties such as low temperature sensitivity and large differential gain. ${ }^{19}$ However, the extension to $1.55 \mu \mathrm{m}$ is not trivial. The

Further author information: (Send correspondence to J.A.G.)

J.A.G.: E-mail: james.gupta@nrc.ca, Telephone: 16139914714

Vertical-Cavity Surface-Emitting Lasers XII, edited by Chun Lei, James K. Guenter,

Proc. of SPIE Vol. 6908, 69080E, (2008) - 0277-786X/08/\$18 - doi: 10.1117/12.763829

Proc. of SPIE Vol. 6908 69080E-1 


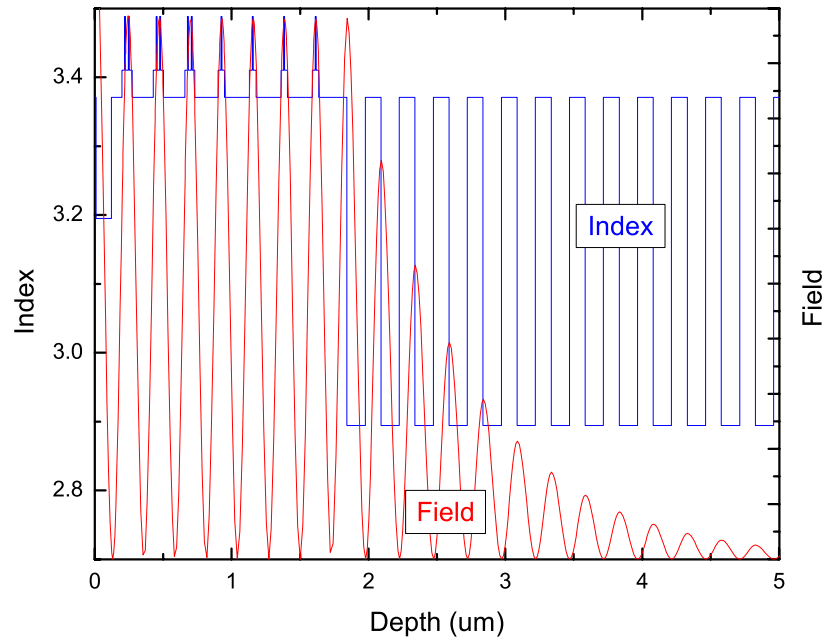

Figure 1. (Color online) Refractive index profile (blue) and electric field intensity (red) through the MBE VECSEL structure described in the text. The sample surface is on the left side of the figure.

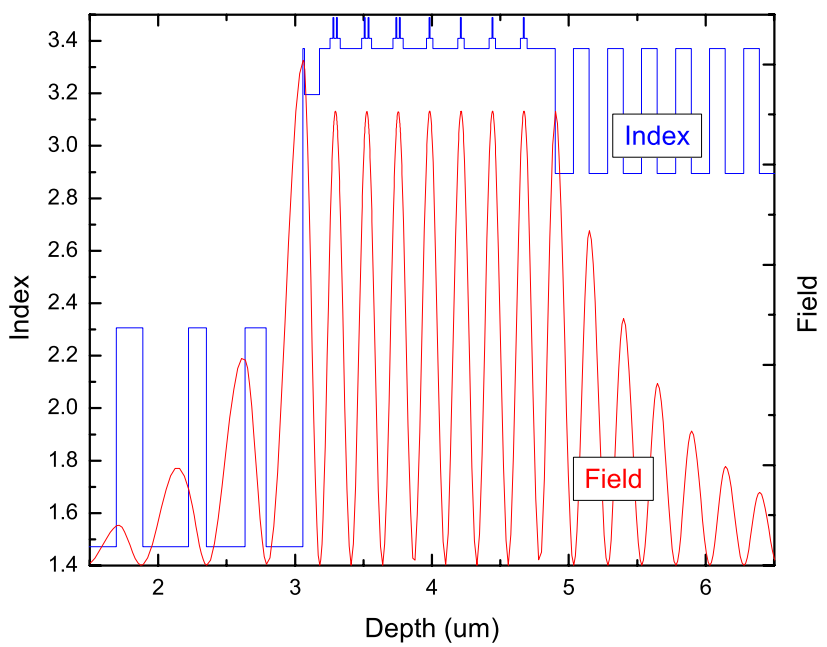

Figure 2. (Color online) Refractive index profile (blue) and electric field intensity (red) through the VCSEL structure including both the MBE layer structure and the dual-band dielectric top mirror, as described in the text.

longer wavelength requires larger indium and nitrogen concentrations, which makes the material more susceptible to phase separation during growth. The addition of Sb to GaInNAs QWs is helpful in reducing this tendency because the $\mathrm{Sb}$ segregates on the growing surface, preventing the formation of In surface clusters. This provides greater flexibility in the MBE growth window, with higher temperatures possible than with GaInNAs. ${ }^{20,21}$ Unlike a pure surfactant, antimony is incorporated in the QWs and provides a small reduction of the bandgap, although its complex incorporation profile leads to a correspondingly complex band alignment. ${ }^{22}$

In the present work, we describe the design, fabrication and characterization of optically-pumped devices operating in the $\mathrm{C}$-band. The devices are based on an epitaxially-grown GaAs-based resonant-periodic gain VECSEL structure containing $10 \mathrm{GaInNAsSb}$ quantum wells. In this case, a VCSEL structure was completed by the deposition of a dielectric top mirror. The temperature performance was studied between 10 and $50{ }^{\circ} \mathrm{C}$ using high-power pulsed pumping. Fabry-Perot analysis was used to study the temporal evolution of the lasing 
wavelength during the pump pulse to provide insight into the pump-induced heating effects. These measurements were performed for chips processed with different rapid thermal annealing temperatures, and hence different cavity-gain detuning. This provides a means of optimizing the detuning to provide the most efficient laser operation.

\section{LASER DESIGN AND FABRICATION}

The laser structure was grown by molecular beam epitaxy (MBE) in a custom V90 system as described elsewhere. ${ }^{21}$ A nominally-exact, semi-insulating GaAs (100) substrate was first prepared with the $600^{\circ} \mathrm{C}$ growth of an undoped GaAs buffer layer and a 31-pair GaAs/AlAs distributed Bragg reflector. During the DBR deposition, accurate in-situ reflectance measurements were used to monitor the layer thicknesses with an SVT Associates In-situ 4000 dual-wavelength $(470 \mathrm{~nm}$ and $950 \mathrm{~nm})$ system. In this way the DBR layer thicknesses were controlled within $1 \%$ of the targets. After the DBR growth, the substrate temperature was lowered to $420^{\circ} \mathrm{C}$ for the growth of a $4-\lambda$, GaAs-based resonant cavity designed for the $\lambda=1.55 \mu \mathrm{m}$ target wavelength. This resonant structure contains 10 GaInNAsSb QWs distributed among the 7 antinodes of the electric field to achieve efficient carrier distribution in VECSEL operation with $808 \mathrm{~nm}$ optical pumping, for which the incident light is nearly completely absorbed $\left(\alpha=13300 \mathrm{~cm}^{-1}\right)$. MBE growth of GaInNAsSb QWs, similar to those employed here, was described in detail recently. ${ }^{21}$ In this case each $7 \mathrm{~nm} \mathrm{Ga}{ }_{0.6} \mathrm{In}_{0.4} \mathrm{~N}_{0.027} \mathrm{As}_{0.9605} \mathrm{Sb}_{0.0125} \mathrm{QW}$ was surrounded by $20 \mathrm{~nm} \mathrm{GaN}_{0.045} \mathrm{As}_{0.955}$ barriers. The entire resonant-cavity active region was grown at the lower temperature so that the quantum wells did not experience any in-situ annealing. Fig. 1 shows the calculated variation of the refractive index and electric field intensity through the VECSEL structure, which does not have a top Bragg mirror.

Several samples from this same MBE epiwafer were further processed into VCSEL devices. As a first step, the pieces were separately ex-situ annealed using a Jipelec Jetfirst 150 rapid thermal annealing (RTA) system. Each piece was annealed for $5 \mathrm{~min}$ in flowing $\mathrm{N}_{2}$ with GaAs proximity capping. The three samples described herein were annealed at temperatures of 725,750 and $775^{\circ} \mathrm{C}$. The VCSEL devices were then formed by the simultaneous deposition of a dual-pump-band $\mathrm{SiO}_{2} / \mathrm{TiO}_{2}$ dielectric top mirror on all three samples. This mirror was designed to allow efficient optical pumping via low reflectivities at $808 \mathrm{~nm}$ and $1064 \mathrm{~nm}$ while providing very high reflectivity at $1.55 \mathrm{um}$. The design employed the proprietary thin film software TFArchitect developed at the National Research Council of Canada. A Spector, Veeco dual ion-beam sputtering system was used for the mirror deposition and the optical constants for the dielectric materials were obtained from ellipsometry analysis of reference layers deposited under similar conditions. In-situ thickness measurements were used and reoptimization was applied to the design to minimize the effects of small thickness variations, as necessary. ${ }^{23}$ Fig. 2 shows the variation of the refractive index and electric field intensity through this hybrid VCSEL structure according to the design.

A comparison between the spectral reflectivities of the MBE VECSEL structure and the hybrid semiconductor/dielectric VCSEL device is shown in Fig. 3. While both structures exhibit a cavity resonance near $1540 \mathrm{~nm}$, the resonance is significantly narrower for the VCSEL. As intended, the VCSEL exhibits a low reflectivity near $1064 \mathrm{~nm}$, on the order of a few percent. The figure also indicates the nonlinear least-squares best-fits to the experimental data, obtained using the Woolam WVASE software package. Extrapolation of the VCSEL best-fit to shorter wavelengths indicates a similarly low reflectivity at $808 \mathrm{~nm}$, as shown. The cavity resonance is at a slightly shorter wavelength than intended because of inaccuracies in our estimations of the GaInNAsSb and GaNAs optical constants. The total thickness of all GaNAs layers in the structure is $340 \mathrm{~nm}$ which is large enough that small errors in index can lead to perceptible shifts in the cavity resonance. For comparison the room-temperature photoluminescence (PL) pectrum of the VECSEL structure with the same RTA conditions is also shown. We note that the PL emission is filtered by the cavity and does not provide an accurate representation of the QW emission spectrum.

\section{LASER CHARACTERIZATION}

The devices were tested on a Cu stage whose temperature was controlled by a circulating water system (though a Peltier could have been used) with no further processing. In future work, performance improvements could 


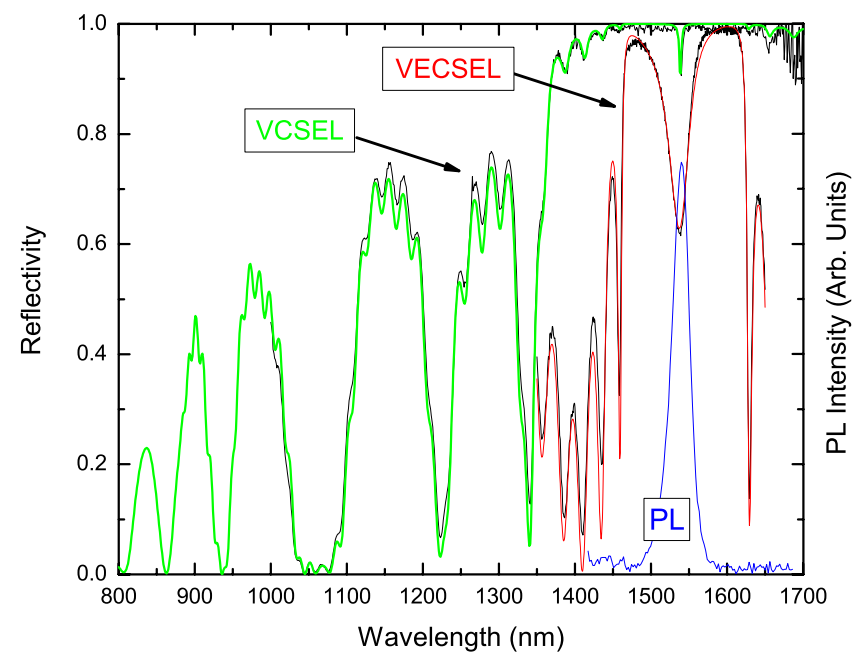

Figure 3. (Color online) Spectral reflectivities of the MBE VECSEL structure and the hybrid VCSEL, both annealed at $775^{\circ} \mathrm{C}$ (black lines). The best fits are shown as red and green lines, respectively. The PL spectrum of the VECSEL structure is also shown (blue line).

be expected through substrate thinning and bonding to high-thermal-conductivity heat sinks, or the use of a capillary-bonded heatspreader. ${ }^{17}$ Surface-normal laser emission of the VCSELs was studied for two separate light sources operating respectively at $850 \mathrm{~nm}$ or $1060 \mathrm{~nm}$, based on the availability of high-power pulsed sources at those wavelengths. The shorter-wavelength pump was a fiber-coupled high-power Perkin-Elmer laser diode, with a rated peak power of $20 \mathrm{~W}$ and a pulse width of $180 \mathrm{~ns}$. Pulsed measurements were made with a $0.18 \%$ duty cycle $(10 \mathrm{kHz})$ at an incidence angle of $30^{\circ}$ and a spot size of $\sim 100 \mu \mathrm{m}$-diameter. The laser power was controlled using a commercial avalanche transistor circuit. Details of a similar pump system were reported elsewhere. ${ }^{24}$ From Fig. 3 we note that the reflectivity at $850 \mathrm{~nm}$ is approximately $12 \%$ at $0^{\circ}$-incidence while the calculated reflectivity for the same structure is $33 \%$ at $30^{\circ}$-incidence. Thus the performance is slightly suboptimal because of the wasted pump power under these conditions. In future work it would be straightforward to optimize the top mirrror design for a selected incident pump angle. The longer wavelength pump source was a Q-switched Nd-YAG laser producing $7 \mathrm{~ns}$-long pulses at a repetition rate of $2 \mathrm{kHz}$ and with maximum pump energy of $17.2 \mathrm{uJ} .{ }^{5}$ In this case the pump power was controlled by attenuation of the beam. In both cases the output power was measured by focusing onto a fast-response InGaAs detector, and the emission spectra were measured using fiber coupling to an optical spectrum analyzer. Power transfer characteristics of the devices were obtained at temperatures between 10 and $50^{\circ} \mathrm{C}$. A photograph of the experimental setup is shown in Fig. 4.

\section{RESULTS AND DISCUSSION}

Preliminary pulsed measurements were made using $1060 \mathrm{~nm}$ excitation, which is absorbed in only the QWs. Temperature-dependent power transfer characteristics are shown in Fig. 5 for the sample annealed at $725^{\circ} \mathrm{C}$. In this figure the pump power was calculated from a measurement of the average power and the pulsed duty cycle. At $20^{\circ} \mathrm{C}$ the device exhibits stimulated emission at $\sim 1535 \mathrm{~nm}$, as shown in the inset. For all samples, the slope efficiency decreases and the threshold pump power increases with increasing heatsink temperature as confirmed in Fig. 6. This behaviour is consistent with material effects due to defect-related or Auger recombination, and to the key laser design issue of gain-cavity detuning. The structure was designed to have a QW emission peak near $1530 \mathrm{~nm}$ after optimized rapid thermal annealing, while the cavity resonance was designed for $1550 \mathrm{~nm}$. This negative detuning was chosen because of a temperature- or pump-induced redshift for the QWs $(\sim 0.5 \mathrm{~nm} / \mathrm{K})$ which is larger than the cavity redshift due to changes in the optical constants of the constituent materials $(\sim 0.1 \mathrm{~nm} / \mathrm{K}) .{ }^{24}$ As shown in Fig. 5 for the sample annealed at $725^{\circ} \mathrm{C}$, the lowest threshold was obtained at the lowest heatsink temperature, suggesting that for this RTA temperature the actual gain-cavity detuning was 


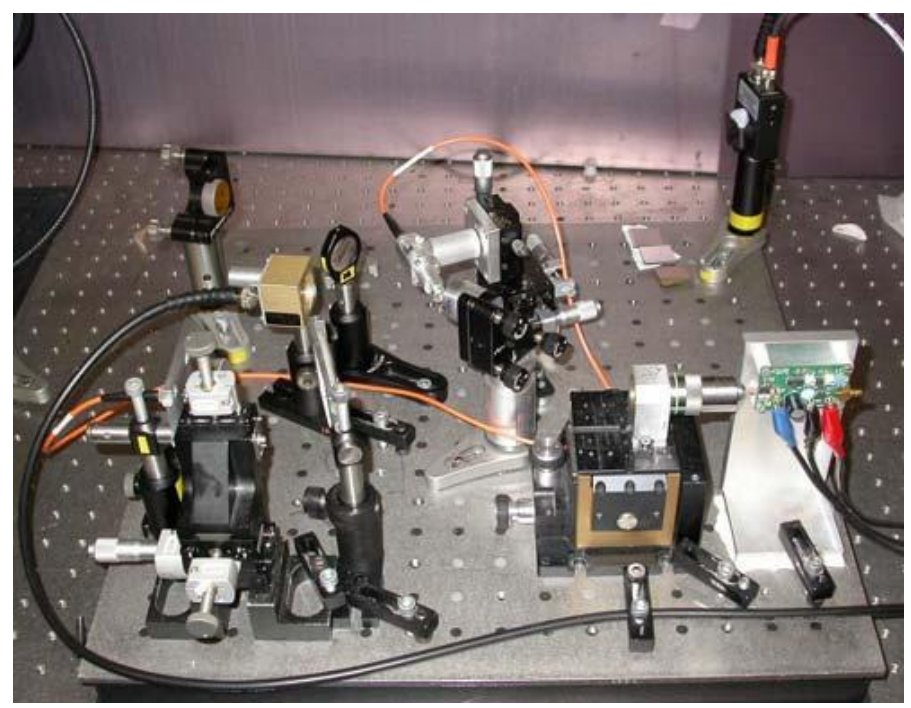

Figure 4. (Color online) Apparatus used for 850nm pumped VCSEL experiment.

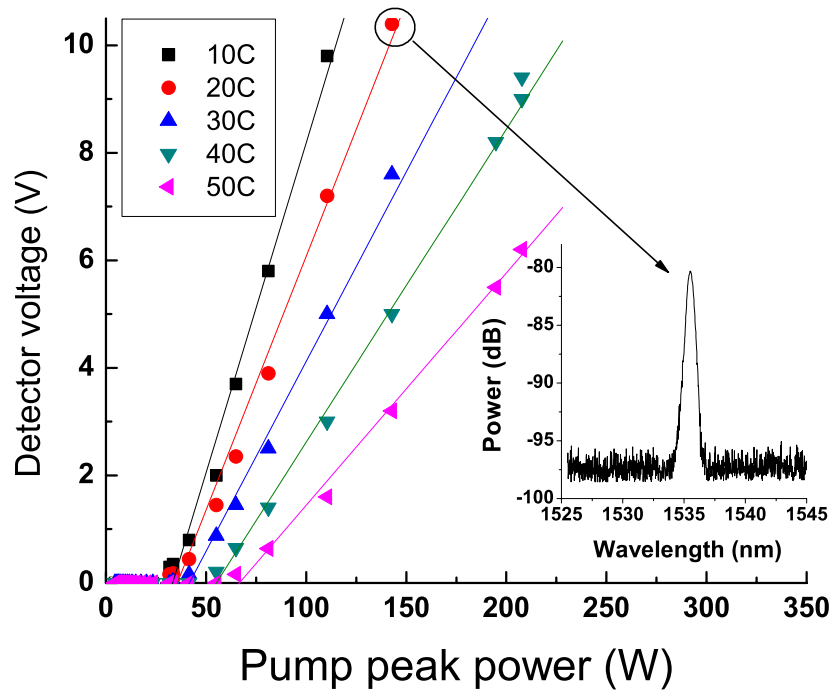

Figure 5. (Color online) Temperature-dependent power transfer characteristics of the VCSEL chip annealed at $725^{\circ} \mathrm{C}$. The inset shows the measured spectrum at $20^{\circ} \mathrm{C}$.

probably slightly lower than optimal. Previous studies have addressed the changes in GaInNAsSb QW emission wavelength with RTA temperature, ${ }^{21}$ indicating that a lower emission wavelength may be obtained at a higher RTA temperature, thus providing a larger gain-cavity detuning. However, Fig. 6 indicate. s that the detuning was too low for even the highest RTA temperature studied here.

To improve pump absorption efficiency, the VCSELs were also tested under $850 \mathrm{~nm}$ pumping, a wavelength condition where the pump is absorbed throughout the active (GaAs) region. The devices were all tested at room temperature. Fig. 7 shows the peak power transfer characteristics for VCSELs with different RTA temperatures. It is interesting to note the clear difference between the threshold peak pump powers for the devices annealed at 750 and $775^{\circ} \mathrm{C}$ compared with the samples annealed at $725^{\circ} \mathrm{C}$. For the lower annealing temperature, the detuning between the QW gain peak and the cavity resonance is smaller and this readily leads to increased threshold.

The temporal evolution of the emitted pulses was also investigated to further our understanding of the operation of these devices. Fig. 8 presents the typical pulse profiles recorded for the $750^{\circ} \mathrm{C}$ annealed sample. It 


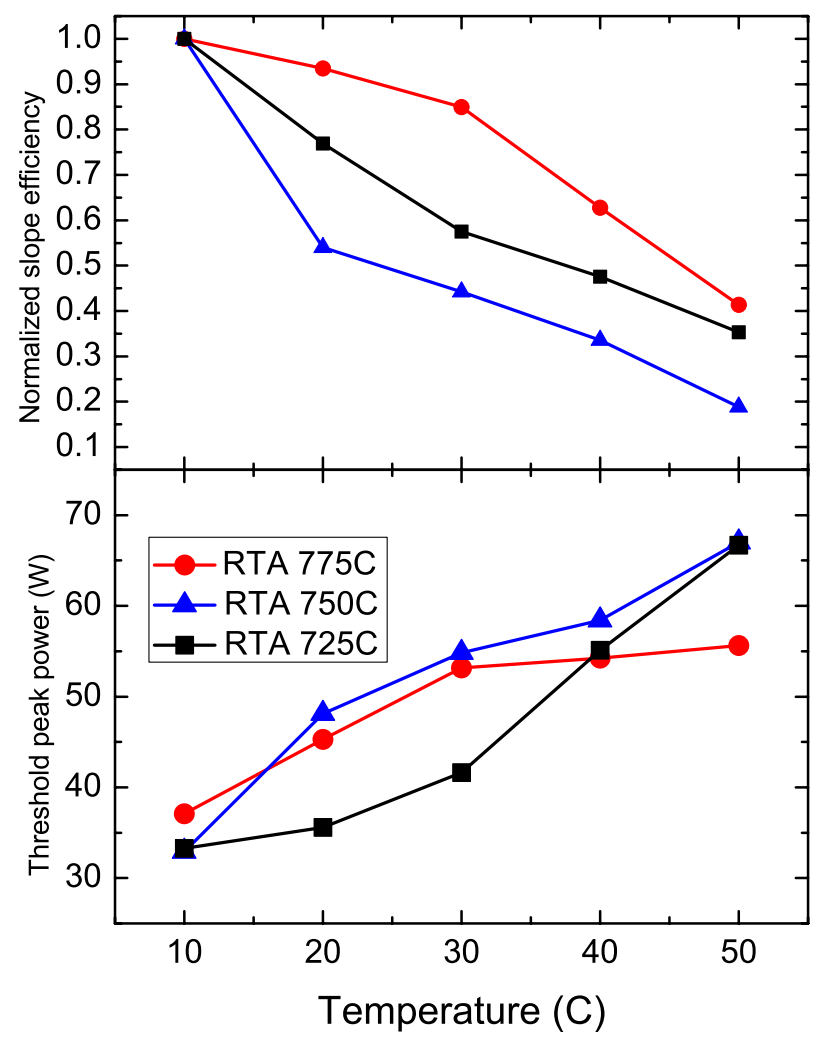

Figure 6. (Color online) Temperature-dependent evolution of threshold (bottom) and normalized VCSEL efficiency (top) under 1060nm pumping.

can be observed that the pulse duration initially increases with pump power, saturates and then decreases. This behaviour is generally shared by all the devices studied here (see summary Fig. 9). Should the devices be able to operate in the continuous-wave (CW) regime, one would expect the evolution of pulse duration to increase until it approximately copies the pump profile $(\sim 180 \mathrm{~ns}$ here). The fact that the saturation and subsequent decrease in the pulse duration occurs at a value much lower than the pump duration indicates that temperature-induced effects (gain-resonance offset and gain shrinkage) control the device emission characteristics to a large degree. The higher threshold and earlier saturation of the pulse duration observed for the $725^{\circ} \mathrm{C}$ annealed sample is consistent with its smaller gain-resonance offset.

In order to confirm this, we also measured the spectral evolution of the emission within the emitted pulse. This was performed by using a Fabry-Perot interferometer as a tunable narrow band filter and recording the temporal evolution for different spectral positions. ${ }^{24}$ Fig. 10 shows the dataset measured for the $750^{\circ} \mathrm{C}$ annealed VCSEL under the maximum pump power condition. As expected, the longest wavelength is reached at the end of the pulse i.e. when the active region has heated up. The laser wavelength was found to shift approximately linearly at a rate of $0.027 \mathrm{~nm} / \mathrm{ns}$ (see Fig. 11) which means that the active region temperature increases at a rate of $0.27 \mathrm{~K} / \mathrm{ns}$ (taking into account the cavity resonance shift of $0.1 \mathrm{~nm} / \mathrm{K}$ ). This suggests that the excursion of active region temperature is less than $27^{\circ} \mathrm{C}$ above room temperature and that, given that edge emitters with the same QWs were shown to have a $\mathrm{T}_{0} \sim 71 \mathrm{~K},{ }^{13}$ the current performance limitation is due to less than optimum gain-cavity offsets. 


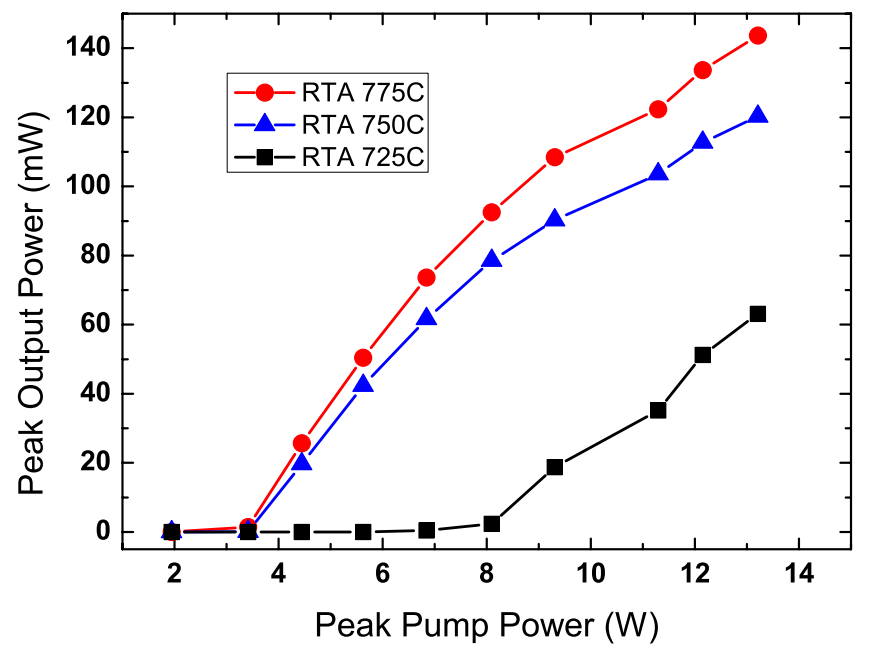

Figure 7. (Color online) Peak power transfer characteristics of VCSEL chips with different RTA temperatures, as indicated.

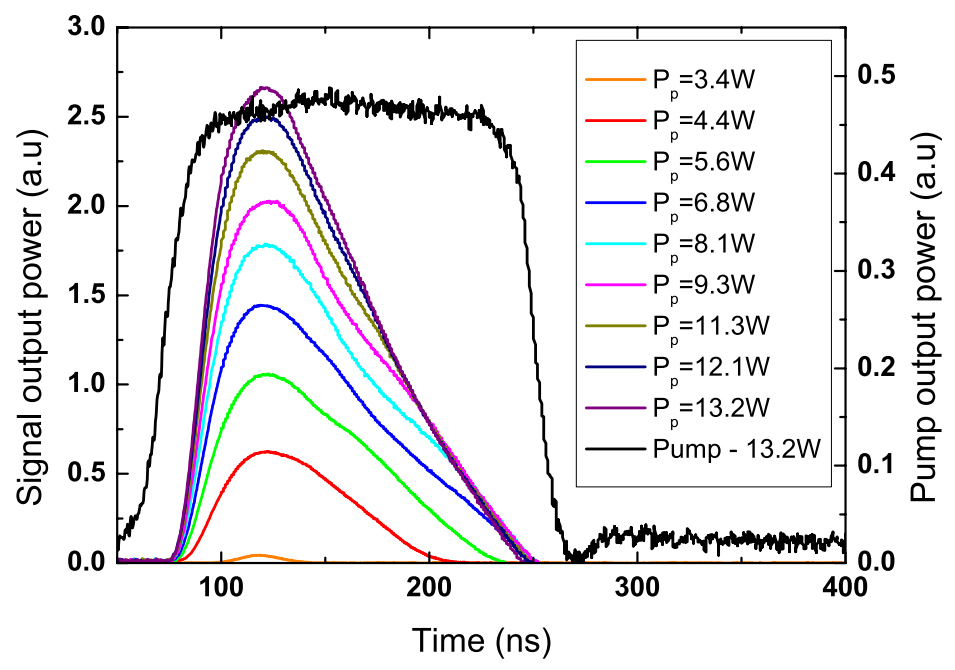

Figure 8. (Color online) Pulse profiles emitted by the VCSEL chip annealed at RTA $750^{\circ} \mathrm{C}$. The pump pulse at full power is shown for reference 


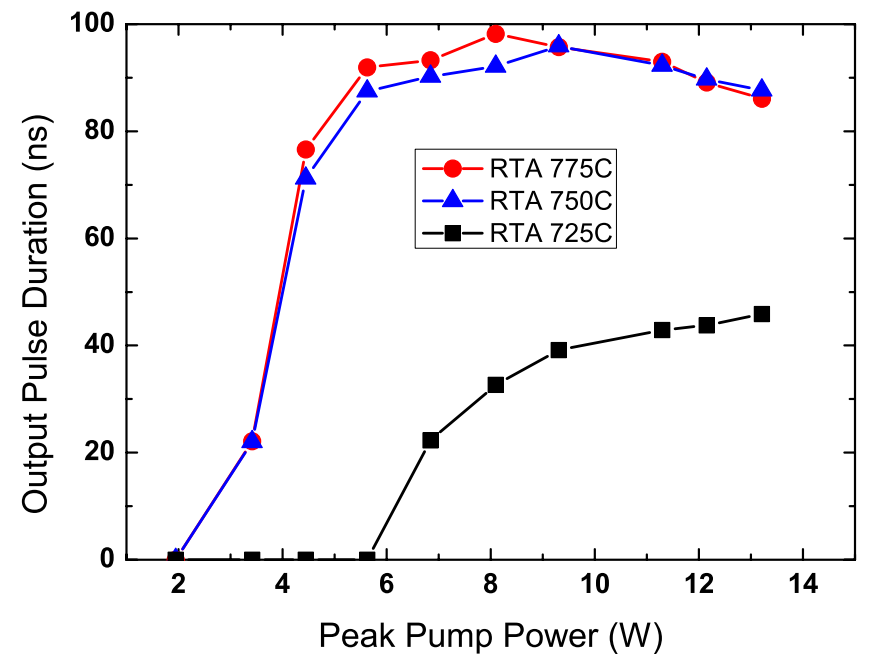

Figure 9. (Color online) Pulse duration emitted by the VCSEL chips with different RTA temperatures, as indicated. Note that the pump pulse varies from 150 to $180 \mathrm{~ns}$ over the same range

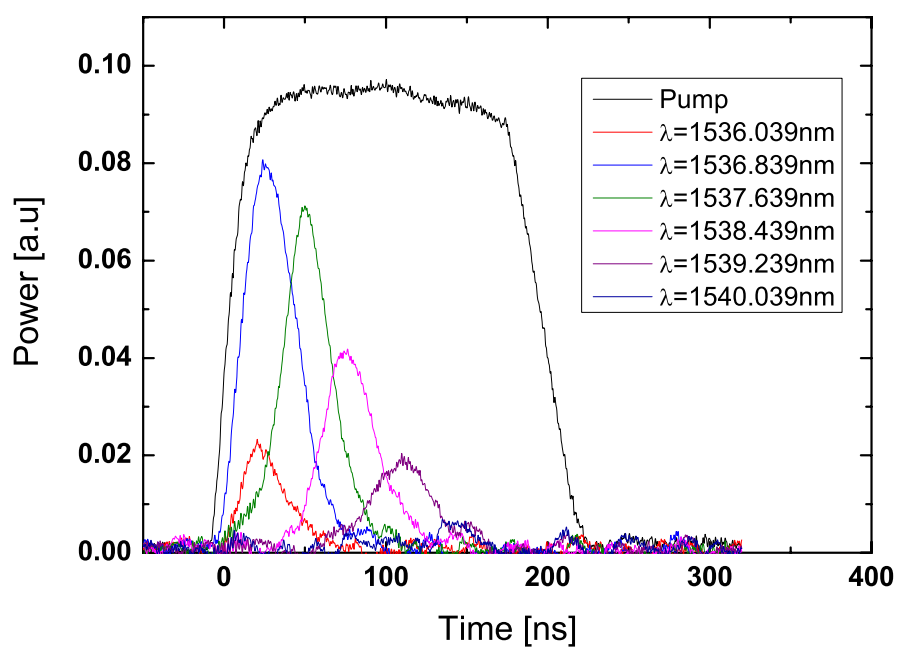

Figure 10. (Color online) Filtered temporal responses of the $750^{\circ} \mathrm{C}$ annealed VCSEL under maximum pumping condition 


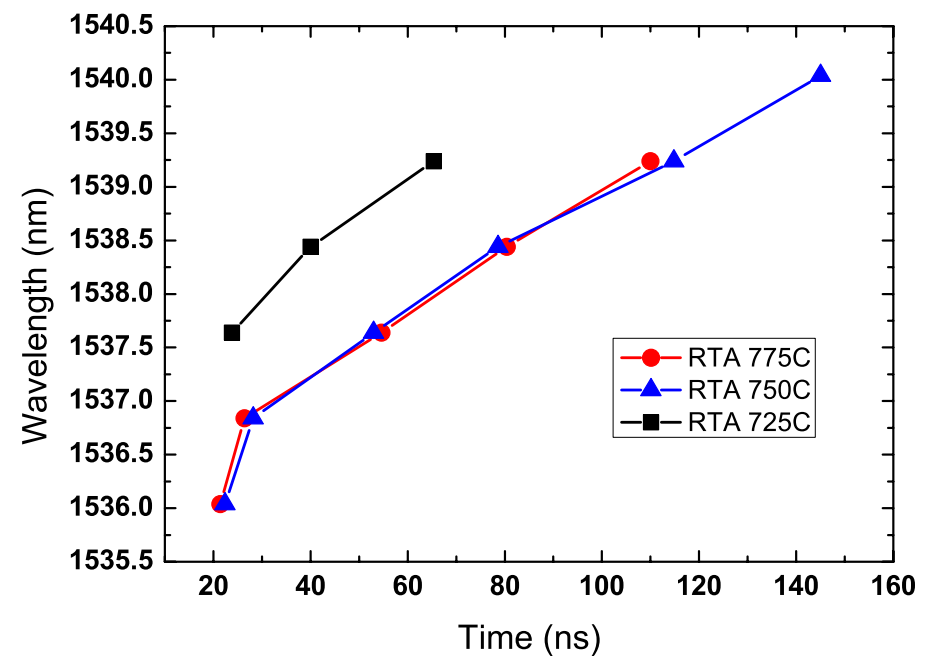

Figure 11. (Color online) Spectral shift of the VCSELs for different annealing temperatures under maximum pumping condition

\section{CONCLUSIONS}

In summary, we have presented a detailed evaluation of optically-pumped $1.55 \mu \mathrm{m}$ GaInNAsSb resonant periodic gain VCSELs grown on GaAs. Temporal and spectral investigations of the pulsed operation of devices with different annealing temperature revealed that that the current laser performance is limited by the gain-cavity offsets. Larger values of detuning can be expected to improve the performance for high-power operation, which can also be assisted by more sophisticated heat extraction schemes. The dual-pump-band dielectric top mirror design has been verified as a useful scheme for both in-well and in-barrier VCSEL pumping, although future design improvements can be expected through consideration of the desired incident pump angles.

\section{ACKNOWLEDGMENTS}

The authors are grateful for the technical support of R. Wang, M. Bresee and helpful discussions with Z.R. Wasilewski.

\section{REFERENCES}

1. C.S. Wang, R. Koda, A.S. Huntington, A.C. Gossard and L.A. Coldren, "> $100 \%$ output differential efficiency $1.55-\mu \mathrm{m}$ VCSELs using submonolayer superlattices digital-alloy multiple-active-regions grown by MBE on InP", J. Cryst. Growth 277, pp. 13-20 (2005).

2. M. Ortsiefer, S. Baydar, K. Windhorn, G. Böhm, J. Rosskopf, R. Shau, E. Rönneberg, W. Hofmann and M.C. Amann, “2.5-mW Single-Mode Operation of 1.55-um Buried Tunnel Junction VCSELs", IEEE Photon. Technol. Lett. 17, pp. 1596-1598 (2005).

3. D. Feezell, D.A. Buell and L.A. Coldren, "InP-Based 1.3-1.6 $\mu \mathrm{m}$ VCSELs With Selectively Etched TunnelJunction Apertures on a Wavelength Flexible Platform", IEEE Photon. Tech. Lett. 17, pp. 2017-2019 (2005).

4. M. Mehta, G. Balakrishnan, S. Huang, A. Khoshakhlagh, A. Jallipalli, P. Patel, M. N. Kutty, L. R. Dawson, and D. L. Huffaker, "GaSb quantum-well-based "buffer-free" vertical light emitting diode monolithically embedded within a GaAs cavity incorporating interfacial misfit arrays", Appl. Phys. Lett. 89, pp. 21111012111103 (2006).

5. N. Laurand, S. Calvez, H.D. Sun, M. Dawson, J.A. Gupta and G.C. Aers, "C-Band Emission from GaInNAsSb VCSEL on GaAs", Electron. Lett. 42, pp. 29-30 (2006). 
6. M.A. Wistey, S.R. Bank, H.B. Yuen, L.L. Goddard, J.S. Harris, "Monolithic GaInNAsSb VCSELs at $1.46 \mu \mathrm{m}$ on GaAs by MBE", Electron. Lett. 39, pp. 1822-1823 (2003).

7. M.A. Wistey, H.P. Bae, H.B. Yuen, E.R. Pickett, L.L. Goddard, J.S. Harris, "GaInNAsSb/GaAs vertical cavity surface emitting lasers at $1534 \mathrm{~nm} "$, Electron. Lett. 42, pp. 282-283 (2006).

8. A. Syrbu, A. Mircea, A. Mereuta, A. Caliman, C.-A. Berseth, G. Suruceanu, V. Takovlev, M. Achtenhagen, A. Rudra, and E. Kapon, "1.5-mW Single-Mode Operation of Wafer-Fused 1550-nm VCSELs", IEEE Photon. Technol. Lett. 16, pp. 1230-1232 (2004).

9. J.A. Gupta, P.J. Barrios, X. Zhang, G. Pakulski and X. Wu, "1.53um GaInNAsSb Laser Diodes grown on GaAs(001)", Electron. Lett. 41, pp. 71-72 (2005).

10. J.A. Gupta, P.J. Barrios, X. Zhang, J. Laponte, G. Pakulski, X. Wu and A. Delâge, "Room-temperature Continuous-wave Operation of GaInNAsSb Laser Diodes at 1.55um", Electron. Lett. 41, pp. 1060-1061 (2005).

11. Z.C. Niu, S.Y. Zhang, H.Q. Ni, D.H. Wu, H. Zhao, H.L. Peng, Y.Q. Xu, S.Y. Li, Z.H. He, Z.W. Ren, Q. Han, X.H. Yang, Y. Du and R.H. Wu, "GaAs-based room-temperature continuous-wave $1.59 \mu \mathrm{m}$ GaInNAsSb single-quantum-well laser diode grown by molecular-beam epitaxy", Appl. Phys. Lett 231121 (2005).

12. S.R. Bank, H.P. Bae, H.B. Yuen, M.A. Wistey, L.L. Goddard, and J.S. Harris, "Room-temperature Continuous-wave $1.55 \mu \mathrm{m}$ GaInNAsSb Laser on GaAs", Electron. Lett. 42, pp.156-157 (2006).

13. J.A. Gupta, P.J. Barrios, J.A. Caballero, D. Poitras, G.C. Aers, G. Pakulski and X. Wu, "Gain and Lifetime

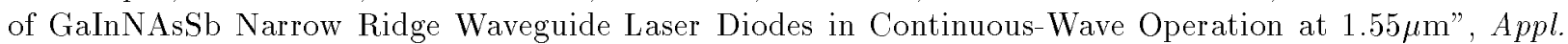
Phys. Lett. 89, 151119 (2006).

14. J.A. Gupta, P.J. Barrios, G. Pakulski, G.C. Aers, J.A. Caballero, D. Poitras and X. Wu, "Properties of GaInNAsSb Narrow Ridge Waveguide Laser Diodes in Continuous-Wave Operation at $1.55 \mu \mathrm{m}$ ", Proc. of SPIE 6485, pp.64850S1-11 (2007).

15. S.R. Bank, H. Bae, L.L. Goddard, H. B. Yuen, M.A. Wistey, R. Kudrawiec and J.S. Harris, "Recent Progress on 1.55-um Dilute-Nitride Lasers", IEEE J. Quantum Electron. 43, pp. 773-785 (2007).

16. H. Shimizu, C. Setiagung, M. Ariga, Y. Ikenaga, K. Kumada, T. Hama, N. Ueda, N. Iwai, and A. Kasukawa, "1.3- $\mu$ m-Range GaInNAsSb/GaAs VCSELs", IEEE J. Sel. Topics Quantum Electron. 9, pp. 1214-1219 (2003).

17. S. Calvez, N. Laurand, H.D. Sun, J. Weda, D. Burns, M.D. Dawson, A. Harkonen, T. Jouhti, M. Pessa, M. Hopkinson, J.A. Gupta, D. Poitras, C.G Leburn, C.T.A. Brown and W. Sibbett, "GaInNAs(Sb) Surface Normal Devices", Phys. Stat. Sol. 203 (2008), and references therein.

18. J. Jewell, L. Graham, M. Crom, K. Maranowski, J. Smith, T. Fanning, "1310nm VCSELs in 1-10Gb/s commercial applications", Proc. of SPIE 6132, pp.24-32 (2006).

19. X. Zhang, J.A. Gupta, P.J. Barrios, G. Pakulski, X. Wu and A. Delage, 'Gain Spectra of 1.3 $\mu \mathrm{m}$ GaInNAs Laser Diodes", J. Vac. Sci. Technol. A 24, pp.787-790 (2006).

20. X. Yang, M.J. Jurkovic, J.B. Heroux and W.I. Wang, "Molecular beam epitaxial growth of InGaAsN:Sb/GaAs quantum wells for long-wavelength semiconductor lasers", Appl. Phys. Lett. 75, pp. 178180 (1999).

21. J.A. Gupta, G.I. Sproule, X. Wu and Z.R. Wasilewski, "Molecular Beam Epitaxy Growth of 1.55 $\mu \mathrm{m}$ GaInNAs(Sb) double quantum wells with bright and narrow photoluminescence", J. Cryst. Growth 291, pp. $86-93(2006)$.

22. H.D. Sun, S. Calvez, M.D. Dawson, J.A. Gupta, G.I. Sproule, X. Wu and Z.R. Wasilewski, "Role of Sb in the growth and optical properties of 1.55 um GaInNSbAs/GaNAs quantum-well structures by molecular-beam epitaxy", Appl. Phys. Lett. 87, pp. 1819081-1819083 (2005).

23. B.T. Sullivan and J.A. Dobrowolski, "Deposition error compensation for optical multilayer coatings. I. Theoretical Description", Applied Optics 31, pp. 3821-3835 (1992).

24. N. Hempler, J.-M. Hopkins, A.J. Kemp, N. Schulz, M. Rattunde, J. Wagner, M.D. Dawson and D. Burns, "Pulsed pumping of semiconductor disk lasers", Optics Express 15, pp. 3247-3256 (2007). 